\title{
Sinonasal teratocarcinosarcoma treated with surgery followed by volumetric modulated radiotherapy: a case report with review of literature
}

\author{
Sarthak Tandon, DNB 1 , Munish Gairola, MD, DNB ${ }^{1}$, Parveen Ahlawat, DNB'1, \\ Kanika Sharma, MD', Soumitra Barik, DMRT ${ }^{1}$, Nishtha Sachdeva, DNB'1, \\ Sunil Pasricha, MD², Apeksha Shenoy, MD³ \\ Departments of ${ }^{1}$ Radiation Oncology, ${ }^{2}$ Pathology, ${ }^{3}$ Radiology and Imaging, Rajiv Gandhi Cancer Institute and \\ Research Centre, New Delhi, India
}

Surgical excision along with use of postoperative radiotherapy forms an integral management of sinonasal teratocarcinosarcoma (SNTCS). However, given the rarity of the tumor, no standardised guidelines, dose, technique and target delineation exist especially in the era of modern radiation delivery techniques. This is a case of 55-year-old male diagnosed as SNTCS treated with radical ethmoidectomy followed by volumetric modulated radiotherapy, showing good local control and acceptable toxicity profile.

Keywords: Intensity-modulated radiotherapy, Volumetric-modulated arc therapy, Malignant teratocarcinosarcoma, Head and neck neoplasms

\section{Introduction}

Sinonasal teratocarcinosarcoma (SNTCS) is a very rare and aggressive neoplasm with lesser than hundred cases being reported in the literature [1-3]. Histologically, it's a unique tumor which contains various elements like teratoma, sarcoma and carcinoma originating from embryonal cell line. Teratocarcinosarcoma was first described by Shanmugaratnam et al. [4], however, the term 'teratocarcinosarcoma' was first coined by Heffner and Hyams [5]. The patient presents most commonly with nasal obstruction, recurrent epistaxis, headache, local pain or visual symptoms. Vague symptoms, presence of air-filled cavity allowing silent growth and rare pathology makes the diagnosis of SNTCS difficult and most of the patients present in advanced stage [2]. With no guidelines available, there is no consensus on the treatment algorithm; however, since most patients fail locally and lymph nodal metastasis is rare; local therapy is considered optimum. Whenever feasible, optimal surgery with complete excision and adequate margins should be first treatment modality. However, most patients present in advanced stage and have infiltrative pattern of spread where complete excision causes unacceptable morbidity. Therefore, radiotherapy becomes an integral part of management of SNTCS, both as primary and adjuvant treatment modality.

Received 27 June 2018, Revised 04 August 2018, Accepted 02 October 2018.

Correspondence: Sarthak Tandon, DNB, Department of Radiation Oncology, Rajiv Gandhi Cancer Institute and Research Centre, Sector-5, Rohini, New Delhi 110085, India. Tel: +91-9871505554, Fax: +91-11-27051037, E-mail: drsarthaktandon@ yahoo.in (http://orcid.org/0000-0002-2319-0391)

(c) This is an Open Access article distributed under the terms of the Creative Commons Attribution Non-Commercial License (http://creativecommons.org/ licenses/by-nc/4.0/) which permits unrestricted non-commercial use, distribution, and reproduction in any medium, provided the original work is properly cited.

www.e-roj.org 


\section{Case Report}

A 55-year-old male presented with complaints of right nasal mass with obstruction. A contrast enhanced magnetic resonance imaging (MRI) of head and neck was done (Fig. 1). Biopsy form the nasal mass revealed teratocarcinosarcoma. The patient underwent radical ethmoidectomy along with reconstruction of medial wall of orbit with PROLENE mesh and left para-median forehead flap using a Lanz incision. Per operative examination revealed a fungating soft tissue mass present in the right ethmoid sinus abutting medial wall of orbit. The soft tissue mass was infiltrating the nasal septa and extending up to contralateral ethmoid. Histopathology report showed an invasive neoplasm with a heterogeneous morphology composed of variable epithelial and mesenchymal components admixed with each other with overall features suggestive of SNTCS (Fig. 2). All resection margins were free however closest soft tissue resection margin was $3 \mathrm{~mm}$ away from the invasive neoplasm.

The patient was evaluated in the Radiation Oncology Clinic and was taken up for adjuvant radiotherapy with intensitymodulated radiotherapy (IMRT) using volumetric modulated arc technique (VMAT). The patient was simulated in the computed tomography (CT) simulator (SOMATOM Sensation Open; Siemens Healthineer, Erlanger, Germany) in supine position and was immobilized with the help of Orfit-ray (Orfit Industries, Wijnegem, Belgium) thermoplastic cast. A slice thickness of $3 \mathrm{~mm}$ was used for target delineation. The Digital

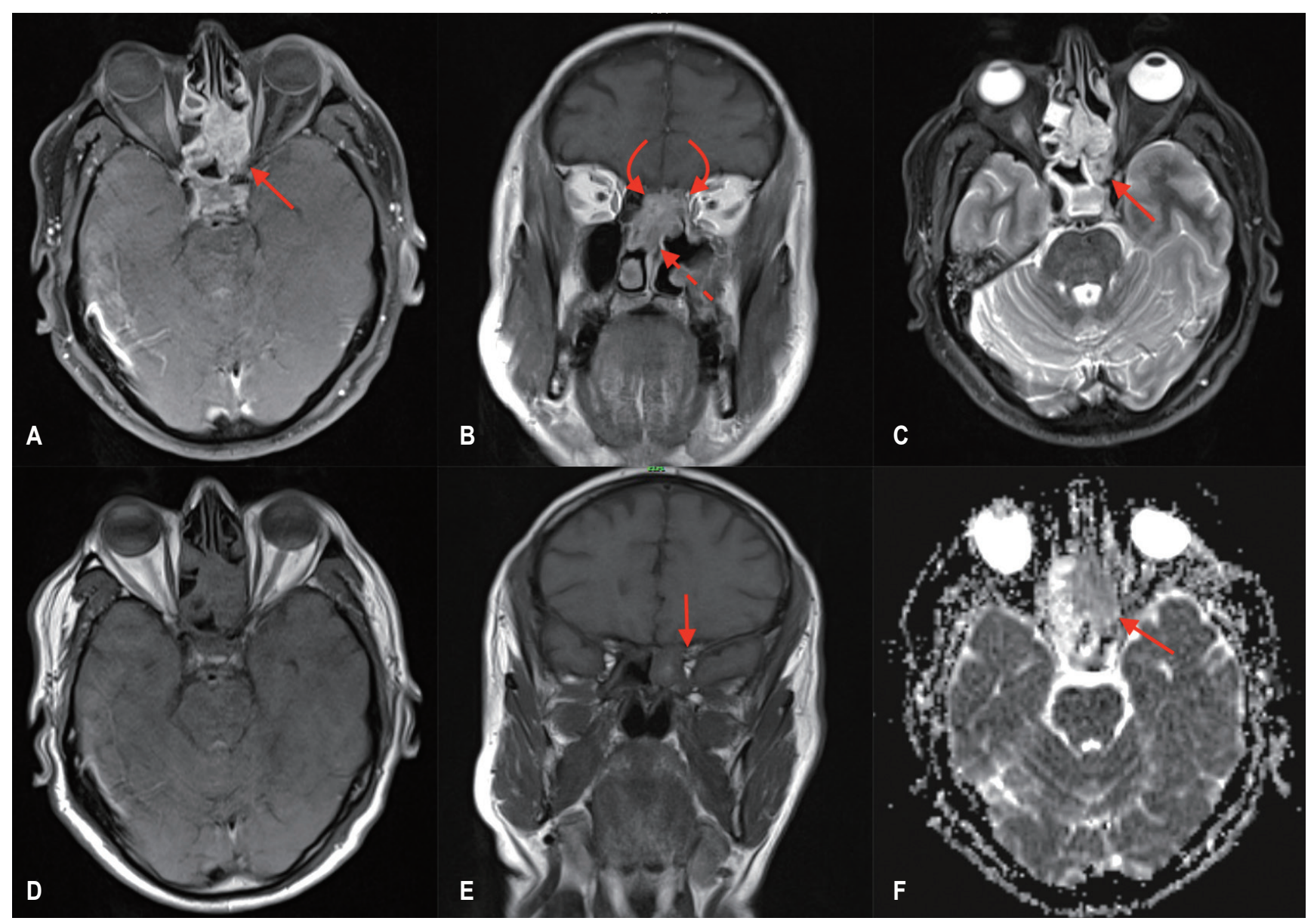

Fig. 1. (A) Fat suppressed post-contrast axial T1 images showing a diffuse homogenously enhancing soft tissue lesion (arrow) in the left posterior ethmoid sinus. (B) Post-contrast coronal T1 images showing a mass involving bilateral cribriform plate (curved arrows) without intracranial extension along with destruction of bony nasal septum (dash arrow). (C) Axial T2 images showing mass extending into left sphenoid sinus posteriorly (arrow). (D) Non-contrast axial T2 images depicting no intraorbital extension. (E) Non-contrast coronal T1 images showing proximity of the mass to left orbital apex and left optic nerve (arrow). (F) Diffusion-weighted ADC map showing dark areas (arrow) on ADC map suggestive of a cellular tumor. ADC, apparent diffusion coefficient. 


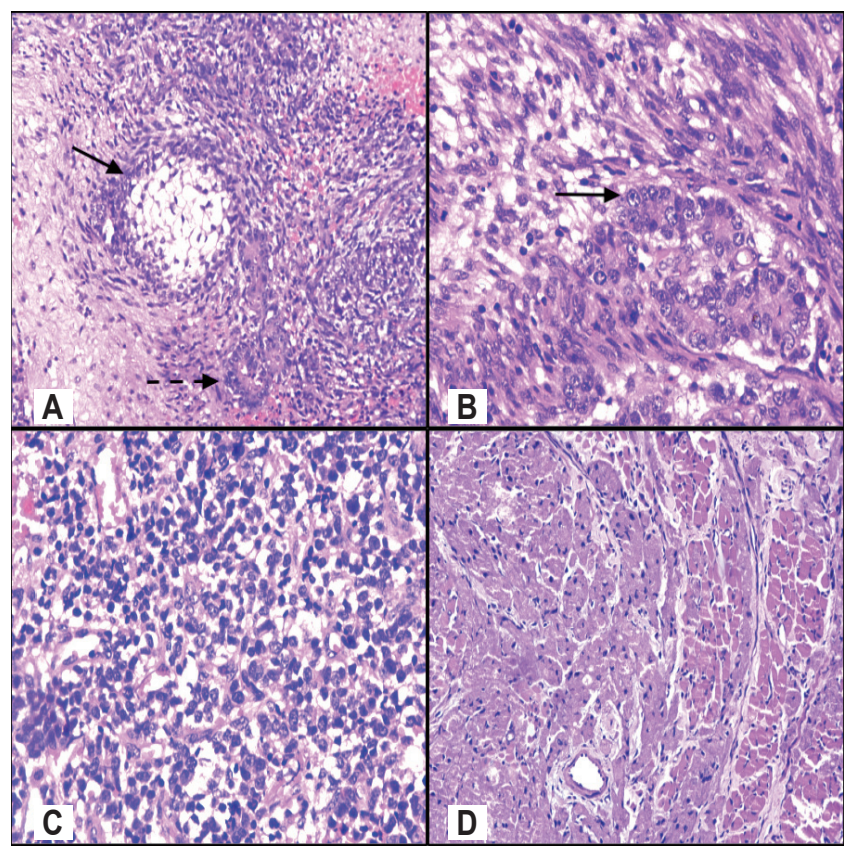

Fig. 2. (A) Intricately admixed malignant glands (bold arrow) and spindle cell stroma along with cutaneous adnexal (dash arrow) structure $(H \& E, \times 100)$. (B) Admixed malignant epithelial (bold arrow) and mesenchymal component $\left(H \& E_{1} \times 400\right)$. (C) Areas of undifferentiated round cell tumor component (H\&E, $\times 200)$. (D) Mesenchymal teratomatous areas showing extensive smooth muscle differentiation $\left(H \& E_{1} \times 200\right)$.

Imaging and Communication in Medicine (DICOM) files was pushed into the Eclipse version 11 (Varian Medical Systems Inc., Palo Alto, CA, USA) where targets were delineated.

For guiding delineation of clinical target volume (CTV), a preoperative gross tumor volume using available MRI scans was generated. CTV included the postoperative bed along with sites of suspicious sub-clinical and microscopic disease extension. A 5-mm isotropic margin was given around CTV to generate planning target volume (PTV). The PTV expansion was limited to $1 \mathrm{~mm}$ near critical structures and bony landmarks. A dose prescription of $60 \mathrm{~Gy} / 30 \mathrm{fx}$ to $95 \%$ of PTV was given. Nodes were not irradiated electively.

VMAT plan was generated using TrueBeam (Varian Medical Systems Inc.), 6-MV photon beams which was optimised using the anisotropic analytic algorithm. The plans used two-arc technique with an arc range from $181^{\circ}$ to $179^{\circ}$ and $179^{\circ}$ to $181^{\circ}$ in clockwise and counter-clockwise direction, respectively. The plan generated had good coverage with 95\% PTV receiving 59.90 Gy with a conformity index and homogeneity index of 1.06 and 0.03 , respectively (Fig. 3). All the organs at risk received acceptable doses (Table 1, Fig. 4).

Acute toxicity assessment was done weekly and at the time of response evaluation as per Common Terminology Criterion of Adverse Events v4.03. The patient tolerated the treatment well and experienced $\leq$ grade 2 acute toxicity (Table 2).

After a follow-up of 12 weeks of completion of radiotherapy, a positron emission tomography-computed tomography (PET-CT) was done which showed no metabolically active or morphologically residual disease suggestive of complete response. The patient was kept on follow-up and was clinically asymptomatic, although at 13 months after completion of radiotherapy, patient developed a right upper deep cervical lymph node. The node was avid on PET scan and was proven positive for malignancy on fine needle cytology. However, the

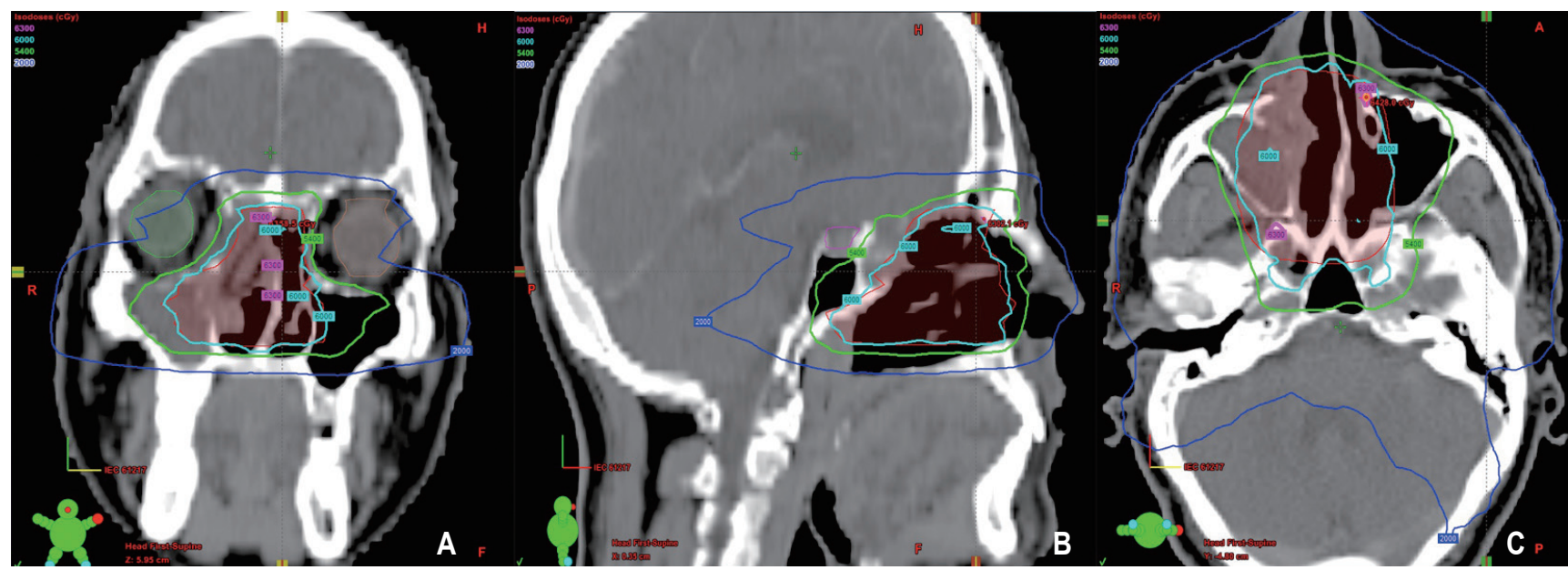

Fig. 3. (A) Coronal, (B) sagittal, and (C) transverse sections showing isodose distribution of radiation planning with good $60 \mathrm{~Gy}$ isodose coverage (cyan) and relative sparing of optic apparatus from $54 \mathrm{~Gy}$ isodose (green). 


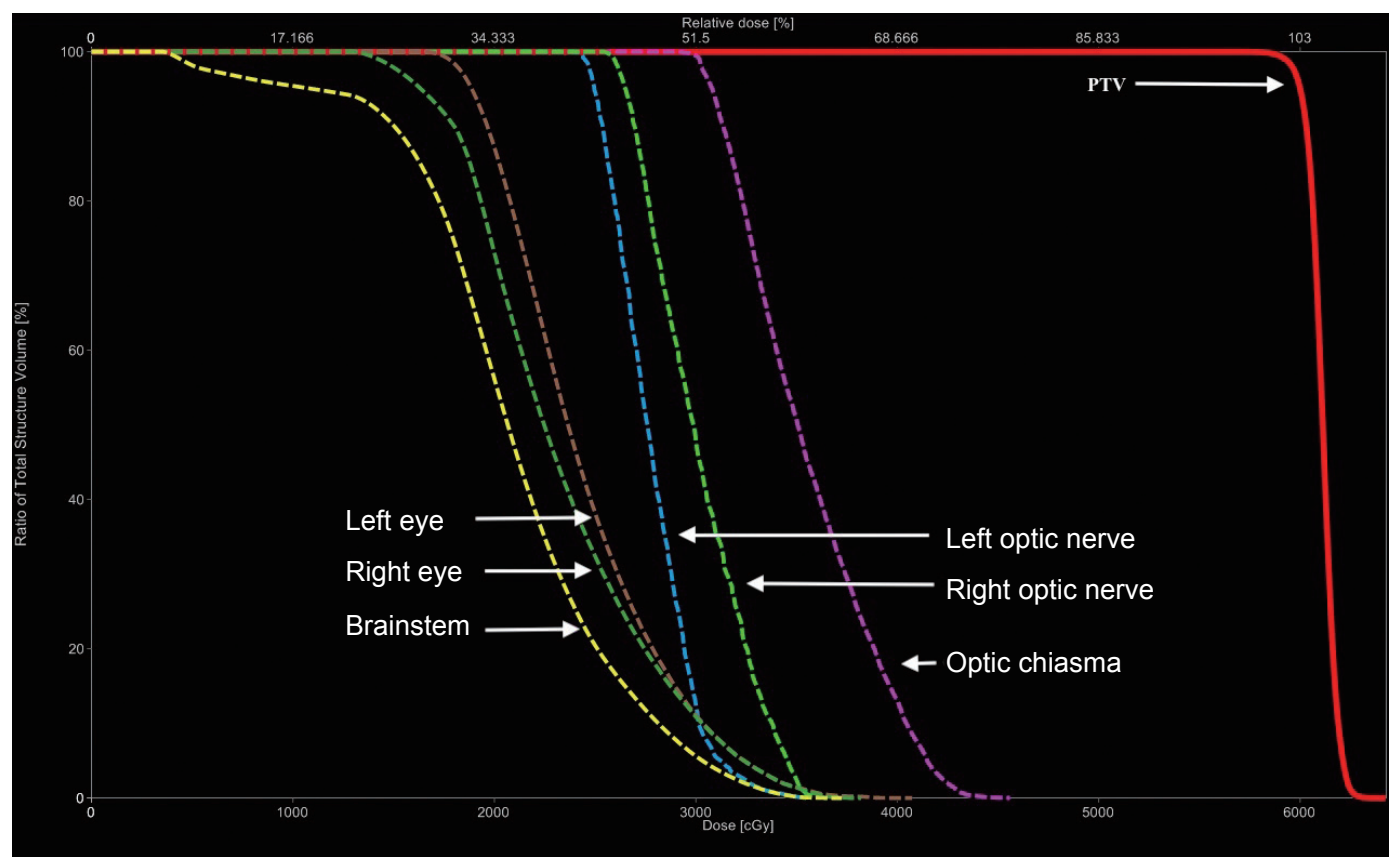

Fig. 4. Cumulative d o s e - volu m e histogram for planning target volume (PTV) and various organs at risk.
Table 1. Organs at risk dosimetric parameters

\begin{tabular}{lcc}
\hline \multicolumn{1}{c}{ Organ } & $D_{\max }(G y)$ & $D_{\text {mean }}(G y)$ \\
\hline Lt. optic nerve & 35.47 & - \\
Rt. optic nerve & 36.42 & - \\
Optic chiasma & 45.58 & - \\
Brainstem & 37.30 & - \\
Lt. eye & 40.77 & - \\
Rt. eye & 38.21 & - \\
Lt. lens & 17.44 & - \\
Rt. lens & 13.28 & - \\
Lt. lacrimal gland & 23.02 & - \\
Rt. lacrimal gland & 19.74 & - \\
Lt. parotid & - & 4.52 \\
Rt. parotid & - & 1.81 \\
Spinal cord & 3.86 & - \\
\hline
\end{tabular}

Table 2. Acute toxicity as per CTCAE v4.03

\begin{tabular}{lc}
\hline \multicolumn{1}{c}{ Acute toxicity } & Grade \\
\hline Mucositis & 2 \\
Radiation dermatitis & 1 \\
Dry eye & 2 \\
Dysphagia & 1 \\
Fatigue & 1 \\
Xerostomia & 1 \\
Dysgeusia & 2 \\
Hoarseness & 1 \\
Oral pain & 1 \\
\hline
\end{tabular}

CTCAE, Common Terminology Criteria of Adverse Events. local control was maintained at the primary site of radiation (Fig. 5). He was successfully salvaged with right modified radical neck dissection and histopathology post dissection was suggestive of teratocarcinosarcoma. Since it was an out of field recurrence and no elective nodal irradiation was given initially, a multispeciality board decision was taken and it was decided to consolidate the draining lymph nodal station with radiation. Retrospectively, it is now considered prudent to electively radiate lymph nodal stations in view of higher chances of local as well regional recurrences. At 18 months follow-up, the patient was disease free.

\section{Discussion}

SNTCS, though very rare, has high predilection for common sites like the nasal cavity, maxillary and ethmoid sinuses; although extranasal sites like nasopharynx, oral cavity and hypopharynx may be involved as well $[6,7]$. Signs and symptoms of nasal obstruction and epistaxis are most common disease presentation. Other symptoms vary from headache, generalised apathy, dizziness and somnolence which may not depend upon the tumor extension [1].

Histopathologically SNTCSs show an irregular glandular or ductal structures within an atypical stromal background. The lesion may comprise of both well-differentiated normal looking epithelia and poorly-differentiated cells representing the malignant component. They may have a glandular pattern 


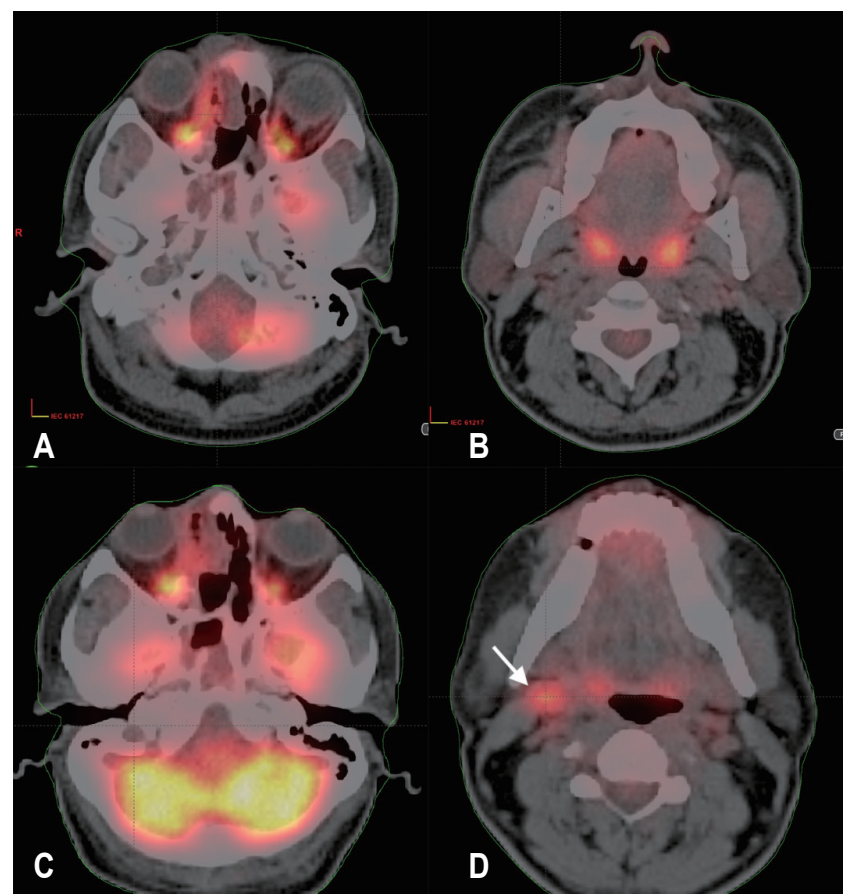

Fig. 5. Transverse sections of PET-CT images at 12 weeks of completion of radiotherapy with no FDG activity in primary $(A)$ or regional lymphatics (B). (C) Transverse sections of PET-CT images at 13 months of completion of radiotherapy with no FDG activity in the primary site but showing FDG activity in the upper deep cervical node (arrow) (D). PET-CT, positron emission tomographycomputed tomography; FDG, 18-fluorodeoxyglucose.

with variable epithelial lining comprising of columnar or cuboidal, ciliated or non-ciliated. Heffner and Hymas [5] described a 'diagnostic clue' where fetal-appearing cells with clear cytoplasm and distinct cytoplasmic membrane representative of squamous component. However, in further literature review [8], these were not reported to be as universal with SNCTS as in the study of Heffner and Hymas [5]. The epithelial component varied from benign glandular epithelium to malignant olfactory epithelium. Apart from the epithelial component, there exists a mesenchymal element comprised of benign to malignant spindle shaped cells in a myxoid stroma. Nests of small blue malignant cells may co-exist and comprise the neuroepithelial element.

SNTCS have a male predilection with most literature [2,9-11] reporting the wide range of median age of presentation as 30 54.7 years. Most studies have reported the clinic-pathological features of SNTCS and are not clear about the prognosis and management of this relatively rare disease. In general consideration, SNTCS is considered to have an aggressive tumor biology with high chances of recurrence and metastasis.
There have two large case series reported till date. On a mean follow-up of 38.9 months, Misra et al. [9] reported 24.4\%, $9.5 \%$, and $6.9 \%$ patients to have local recurrences, metastatic disease, and both, respectively. Budrukkar et al. [2] reported $68.7 \%$ failure rate with $56.3 \%$ patient failing locoregionally with a median follow-up of 34 months and median time of recurrence of 7 months. The treatment algorithm largely remains unclear primarily due to lack of any larger clinical series and trial. However, most series have reported the use of surgery and radiotherapy.

The role of systemic therapy remains unclear; however, there is limited evidence to support the role of adjuvant chemotherapy. Misra et al. [9] reported survival for various subsets of treatment combination of chemotherapy, radiotherapy and surgery albeit with low number in most of the groups. Fifty-one patients were treated with surgery and radiation and had a survival of $56.5 \%$ at a follow-up of 45.4 months. In comparison, only 10 patients were treated with surgery, radiation and chemotherapy had a survival of $88.8 \%$ at a follow-up of 32.1 months. With such gross differences between the groups, it becomes difficult to arrive at a meaningful conclusion regarding the role of chemotherapy.

An institutional study has reported the use of neoadjuvant chemotherapy [12] and tailored chemotherapy [13] (depending upon the type of predominant histology in the postoperative specimen) to avoid extensive craniofacial resection and positive margins. These protocols may have theoretical advantage in the management of SNTCS, however, lack of larger studies may confound in validation of these management algorithms. Platinum is one of the commonest drug used in most series; either in concurrence with radiation or another chemotherapy drug.

Radiotherapy plays an integral role in the management of SNTCS. Most series that have been published are either from the two-dimensional conventional era or have used threedimensional conformal radiotherapy with variable dose ranging between 10 and $70 \mathrm{~Gy}$; most commonly between 54 and 60 Gy. The dose was primarily restricted due to the infiltrative pattern and neighbouring critical structures. Although, in the era of IMRT, higher doses can be delivered to primary tumor volume and controlling doses to the neighbouring critical structures simultaneously. Safety and efficacy for IMRT has already established for sinonasal malignancy to an extent that a phase 3 trial has showed survival advantage of using IMRT in nasopharyngeal tumor in comparison to conventional radiotherapy. However, whether this clinical advantage of IMRT can be extrapolated to SNTCS is a matter of debate as there is 
no trial suggesting the use of IMRT in SNCTS primarily because of the rarity of the tumor. Reports using IMRT in SNTCS with a dose prescription 60-66 Gy, reported local control ranging between 2 and 3.5 years [14-16].

Extensive literature research revealed only handful of case reports using IMRT in SNTCS and none suggesting the use of VMAT. However, using VMAT for nasal cavity and paranasal sinus tumors is well documented and has dosimetric superiority [17-21]. The dosimetric superiority has been reported both in terms of PTV coverage $[17,20]$, reducing OARs doses $[17,18,20]$ and decreasing delivery times and monitor units [18-20]. The three parameters (continuous gantry rotation with variable speed, simultaneous microleaf collimator movement, and continuous dose rate variation) used in VMAT planning allows for greater degree of freedom for optimisation in comparison to IMRT. However, reports also assert caution while using VMAT for paranasal and nasal cavity tumors where beam angle selection is limited in view of location of the tumor. This might lead to inadvertent low-dose spills into OARs especially lens [21].

Review of literature did not reveal any consensus regarding the use of elective nodal irradiation primarily because of the non-uniformity of the reported data regarding radiation fields and technique. Six reports $[1,2,6,11,14,22]$ have commented on the use or disuse of elective nodal irradiation. Of 4 studies reporting no treatment to neck, 2 studies $[2,6]$ have documented regional lymph node failures. With 2 studies $[1,11]$ reporting elective nodal irradiation, none had reported locoregional failure. Although, it becomes difficult to analyse the need of elective nodal irradiation with the current evidences available, the use of elective nodal radiotherapy may be justified in SNTCS which has high probability of locoregional failure.

In conclusion, the case demonstrates that IMRT using VMAT is a feasible and safe method to deliver radiotherapy in a case of SNTCS. It also highlights the importance of elective nodal irradiation in the absence of radiologically or clinically palpable nodes. Larger studies would be required to streamline the management of SNTCS, however, given the rarity of the tumor it seems unlikely in the near future.

\section{Conflict of Interest}

No potential conflict of interest relevant to this article was reported.

\section{References}

1. Agrawal N, Chintagumpala M, Hicks J, Eldin K, Paulino AC. Sinonasal teratocarcinosarcoma in an adolescent male. J Pediatr Hematol Oncol 2012;34:e304-7.

2. Budrukkar A, Agarwal JP, Kane $S$, et al. Management and clinical outcome of sinonasal teratocarcinosarcoma: single institution experience. J Laryngol Otol 2010;124:739-43.

3. Naik SM, Nanjundappa RH, Srihariprasad KS, Gupta S, Rao CR. Sinonasal teratocarcinosarcoma: a rare clinical entity managed by medial maxillectomy and adjuvant chemoradiation. Clin Rhinol Int J 2012;5:118-22.

4. Shanmugaratnam K, Kunaratnam N, Chia KB, Chiang GS, Sinniah R. Teratoid carcinosarcoma of the paranasal sinuses. Pathology 1983;15:413-9.

5. Heffner DK, Hyams VJ. Teratocarcinosarcoma (malignant teratoma?) of the nasal cavity and paranasal sinuses $A$ clinicopathologic study of 20 cases. Cancer 1984;53:2140-54.

6. Smith SL, Hessel AC, Luna MA, Malpica A, Rosenthal DI, ElNaggar AK. Sinonasal teratocarcinosarcoma of the head and neck: a report of 10 patients treated at a single institution and comparison with reported series. Arch Otolaryngol Head Neck Surg 2008;134:592-5

7. Carrizo F, Pineda-Daboin K, Neto AG, Luna MA. Pharyngeal teratocarcinosarcoma: review of the literature and report of two cases. Ann Diagn Pathol 2006;10:339-42.

8. Pai SA, Naresh KN, Masih K, Ramarao C, Borges AM. Teratocarcinosarcoma of the paranasal sinuses: a clinicopathologic and immunohistochemical study. Hum Pathol 1998;29:718-22.

9. Misra P, Husain O, Svider PF, Sanghvi S, Liu JK, Eloy JA. Management of sinonasal teratocarcinosarcoma: a systematic review. Am J Otolaryngol 2014;35:5-11.

10. Fatima SS, Minhas K, Din NU, Fatima S, Ahmed A, Ahmad Z. Sinonasal teratocarcinosarcoma: a clinicopathologic and immunohistochemical study of 6 cases. Ann Diagn Pathol 2013;17:313-8.

11. Wei $S$, Carroll W, Lazenby A, Bell W, Lopez R, Said-Al-Naief $N$. Sinonasal teratocarcinosarcoma: report of a case with review of literature and treatment outcome. Ann Diagn Pathol 2008;12:415-25.

12. Joshi A, Noronha V, Sharma M, et al. Neoadjuvant chemotherapy in advanced sinonasal teratocarcinosarcoma with intracranial extension: report of two cases with literature review. J Cancer Res Ther 2015;11:1003-5.

13. Singh ND, Sen K, Sharma A, Bharadwaj M. Sinonasal teratocarcinosarcoma: is minimally invasive resection 
followed by adjuvant histology directed chemo-radiation a better alternative to radical excision? Case report. Int J Otorhinolaryngol Head Neck Surg 2017;3:452-4.

14. Tokunaga $T$, Sunaga $H$, Kimura $Y$, Tsuda G, Fujieda S. A case of sinonasal teratocarcinosarcoma treated with surgery and post-operative intensity-modulated radiotherapy (IMRT). Auris Nasus Larynx 2012;39:641-5.

15. Peng G, Ke Y, Wang T, Feng Y, Li Y, Wu G. Intensity-modulated radiotherapy for sinonasal teratocarcinosarcoma. J Huazhong Univ Sci Technolog Med Sci 2011;31:857-60.

16. Foong YC, Murdolo V, Naiman N, Hepner L, Awad R. Sinonasal teratocarcinosarcoma: a case report. J Med Case Rep 2017;11:167.

17. Sankaralingam $M$, Glegg $M$, Smith $S$, James $A$, Rizwanullah $M$. Quantitative comparison of volumetric modulated arc therapy and intensity modulated radiotherapy plan quality in sinonasal cancer. J Med Phys 2012;37:8-13.

18. Nguyen $K$, Cummings $D$, Lanza VC, et al. A dosimetric comparative study: volumetric modulated arc therapy vs intensity-modulated radiation therapy in the treatment of nasal cavity carcinomas. Med Dosim 2013;38:225-32.

19. Studenski MT, Bar-Ad V, Siglin J, et al. Clinical experience transitioning from IMRT to VMAT for head and neck cancer. Med Dosim 2013;38:171-5.

20. Jeong $Y$, Lee SW, Kwak J, et al. A dosimetric comparison of volumetric modulated arc therapy (VMAT) and non-coplanar intensity modulated radiotherapy (IMRT) for nasal cavity and paranasal sinus cancer. Radiat Oncol 2014;9:193.

21. Guckenberger M, Richter $A$, Krieger T, Wilbert J, Baier $K$, Flentje $M$. Is a single arc sufficient in volumetric-modulated arc therapy (VMAT) for complex-shaped target volumes? Radiother Oncol 2009;93:259-65.

22. Nitsche M, Hermann RM, Christiansen H, Berger J, Pradier 0 . Rationale for individualized therapy in Sinonasal Teratocarcinosarcoma (SNTC): case report. Onkologie 2005;28:653-6. 